\title{
Histomorphological changes in the cornea of the rat following monocular eyelid closure
}

\author{
Faith Bonareri Aseta, Philip Maseghe Mwachaka, Paul Ochieng Odula, Adel Kamel Malek \\ Department of Human Anatomy, School of Medicine, Nairobi University, Nairobi, Kenya
}

\begin{abstract}
Objectives: The aim of this study was to describe structural changes in the cornea of the rat after monocular eyelid closure. Methods: Twenty-six Rattus norvegicus male rats aged three months were used. The rats were randomly assigned into baseline (2), experimental (16) and control (8) groups. Unilateral eyelid closure was performed on the experimental animals by suture tarsorrhaphy. At experiment days 5, 10, 15 and 20, four rats from the experimental group and two rats from the control group were euthanized, their eyeballs harvested, and routine processing was done for paraffin embedding, sectioning and Masson's trichrome staining. The photomicrographs were taken using a digital photomicroscope.

Results: In the closed eyes, there was a time-dependent reduction in the stratification of the corneal epithelium with subsequent disintegration, and an increase in distribution of stromal keratocytes while the corneal endothelial cells showed slight enlargement from squamous shape. The contralateral and control eyes did not exhibit any significant changes through the experimental period.

Conclusion: Monocular eyelid closure causes structural changes in the corneal epithelium, stroma and endothelium of the tarsorrhaphy eye. Therefore, tarsorrhaphy should not be prolonged due to risk of corneal diseases and diminution of vision as a result of the structural changes.
\end{abstract}

Keywords: cornea; eyelid closure; rat; tarsorrhaphy

Anatomy 2016;10(2):87-93 (02016 Turkish Society of Anatomy and Clinical Anatomy (TSACA)

\section{Introduction}

Corneal avascularity is important for its transparency. ${ }^{[1]}$ However, this presents a physiological challenge for the supply of metabolic factors, especially oxygen. ${ }^{[2]}$ Therefore, corneal oxygenation is by non-vascular sources including the aqueous humor, conjuctival tear film, and direct atmospheric oxygen as the most significant source in open eye conditions. ${ }^{[3]}$ Eyelid closure deprives the cornea of atmospheric oxygen along with light. ${ }^{[4]}$

Clinically, eyelid closure is performed in several conditions including corneal epithelial defects, exposure keratopathy, neurotrophic ulcers, chemical burns, diabetic eye disease, Graves' disease and Bell's palsy. ${ }^{[5]}$ When prolonged, eyelid closure is a risk factor for complications such as recurrent corneal erosion, corneal de-epithelialization and keratitis which may affect vision. ${ }^{[5,6]}$ There is, however, paucity of information on the anatomical changes in the individual layers of the cornea with eyelid closure.
This study therefore set out to investigate the histological changes in the cornea following complete unilateral eyelid closure, using a rat model. This knowledge may contribute to clinical practice in the management of eye diseases using complete tarsorrhaphy, and to the understanding of importance of atmospheric oxygen in maintaining the anatomy and functioning of the cornea.

\section{Materials and Methods}

The ethical approval to carry out the study was granted by the Biosafety, Animal Care and Use Committee of Nairobi University, Nairobi, Kenya (Ethical approval number: FVMBAUEC/2015/79). As the study model, male rats of Rattus norvegicus species were used because corneal structure in rats exhibits a similar histological organization to that of human beings. ${ }^{[7]}$ In addition, Rattus norvegicus is widely used in experimental eye research as an animal model for human eye disease. ${ }^{[8]}$ 
The animals were about three months old, since corneal maturation is complete by this age. The sample size was obtained using convenient sampling. Male as opposed to female rats were used, since the cornea has receptors for estrogen, a modulating factor in its biomechanical properties. ${ }^{[9]}$ The rats were kept in cages with sawdust bedding that was regularly changed. Rat pellets and water were provided ad libitum.

Two rats were used for the baseline (day 0). The remaining twenty four animals were randomly assigned to experimental and control groups (16 experimentals, 8 controls). Unilateral eyelid closure was performed on the experimental animals by suture tarsorrhaphy, eight animals on the right and eight on the left eye. The animals were anesthetized using 5\% inhalational halothane following which four horizontal mattress stitches were made on the external lamina of the upper and lower eyelid margins using polypropylene monofilament 5-0 sutures. The experimental animals were observed daily to ensure the sutures were intact. Combined indomethacin/gentamycin eye drops were administered twice daily post-operatively to minimize pain and prevent infections. Animals with signs of post-procedural pain such as reduced activity, abnormal posture, decreased food and water intake and increased aggression were further given subcutaneous ketoprofen injection at a dose of $5 \mathrm{mg} / \mathrm{kg}$. No animal developed suture dehiscence. The control animals did not undergo tarsorrhaphy on either eye.

At experiment days 5, 10, 15 and 20, four rats from the experimental group and two rats from the control group were euthanized, perfused with normal and formal saline, their eyeballs harvested and processed for paraffin-embedding and sectioning. The rats' eyeballs were fixed in $10 \%$ formalin for twelve hours. This was followed by dehydration in increasing grades of alcohol (70\% up to absolute alcohol) at one hour intervals, and clearing in toluene. Thereafter, a three millimeter slit was made at one side of the eyeball to allow for wax infiltration in the memmert oven, which took eighteen hours. The eyeballs were embedded in paraffin wax and oriented for transverse sectioning. After cooling, the embedded tissues were blocked using wooden blocks and then serially cut into $7 \mu \mathrm{m}$ sections using a microtome. The transverse sections were made up to the level of the optic nerve from where five ribbons were obtained. Ten $7 \mu \mathrm{m}$ sections were randomly obtained from the five ribbons, floated on a $60{ }^{\circ} \mathrm{C}$ water bath and picked on a glass slide, then dried in an oven for 12 hours. Thereafter, staining was performed using Masson's trichrome method. Photomicrographs of the sections were taken using a digital camera (Canon Powershot A640, $12 \mathrm{mp}$, Beijing, China) mounted on a photomicroscope (Carl Zeiss, Axiostar Plus Microimaging, Jena, Germany). The morphological changes in each of the corneal layers were described.

\section{Results}

The cornea comprised a stratified squamous non-keratinized epithelium, stroma and endothelium for all the studied animals. The changes in the Bowman's membrane and the Descemet's membrane could not be clearly delineated with light microscopy. There were no noticeable side differences in the morphology of the cornea. At baseline, the epithelium contained four to five cell layers resting on a basement membrane. The deep cell layers contained ovoid nuclei while the superficial cell layers comprised flattened nuclei. The stroma contained densely packed parallel collagen bundles that appeared wavy with interspersed fibroblast-like cells, known as keratocytes. The nuclei of the keratocytes were flat and elongated. The stroma occupied most of the corneal thickness. The endothelium was made up of simple squamous cells whose nuclei were sparsely placed from each other (Figure 1).

Table 1 summarizes the histomorphological changes observed, in comparison to the control group. In the tarsorrhaphy eyes, there was gradual loss of the superficial cells of the epithelium with increasing periods of monocular deprivation (Figures 2a-d). At day 20, vacuolations and disintegration within the superficial layers were noted, leaving only the basal layer (Figure 2d). With increasing period of deprivation, the keratocytes within the parallel collagen fibers of the stroma increased in dis-

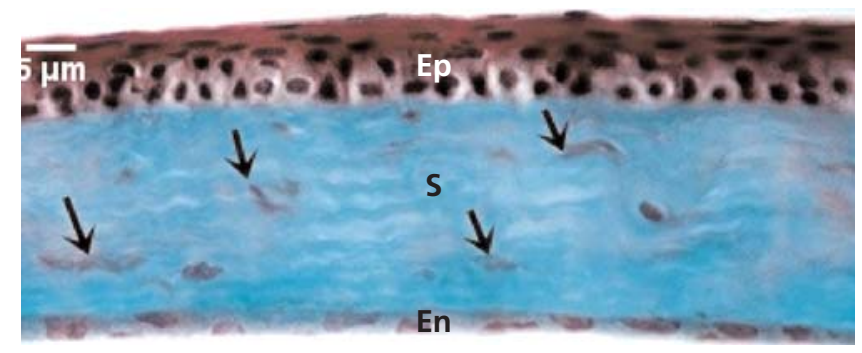

Figure 1. The photomicrograph of the cornea at baseline. The epithelium (Ep) is stratified with basal cells having ovoid nuclei while the superficial ones contain flattened nuclei. The stroma (S) comprises wavy collagen fibers with scattered keratocytes (arrows). The endothelium (En) is composed of a simple squamous epithelium (Masson's trichrome stain, $\times 400$ ). [Color figure can be viewed in the online issue, which is available at www.anatomy.org.tr] 
Table 1

Time-dependent histological changes in corneal layers with increasing period of monocular deprivation.

\begin{tabular}{llll}
\hline Corneal layers & Tarsorrhaphy eyes & Contralateral open and control eyes & Stratified epithelium similar to baseline \\
\hline Epithelium & $\begin{array}{l}\text { Gradual decrease in stratification (from baseline to day 20) } \\
\text { Vacuolation and disintegration of superficial layers; basal layer intact (day 20) }\end{array}$ & Sparse distribution of keratocytes, similar to baseline \\
\hline Stroma & $\begin{array}{l}\text { Gradual increase in distribution of corneal keratocytes (from baseline to day 20) } \\
\text { Change in shape of keratocytes from flat to rounded appearance (day 15-20) }\end{array}$ & Squamous cells, similar to baseline & Slight enlargement of endothelial cells (day 10 to day 20) \\
\hline Endothelium & &
\end{tabular}

tribution (Figures 2a-d), and appeared less elongated and more rounded (Figure $\mathbf{2 d}$ ). The endothelium was simple squamous for all the days but the nuclei appeared slightly larger from day 10 onwards (Figures $\mathbf{2 b - d}$ ).

In the non-tarsorrhaphy (contralateral) eyes, the corneal epithelium was stratified squamous non-keratinized on all the experimental days (Figures 3a-d). There were no obvious changes in the epithelium with increasing periods of monocular eyelid closure. The corneal stroma comprised densely packed collagen fiber bundles with few scattered keratocytes containing elongated nuclei. No obvious changes were noted in the endothelium.

Similarly in the control eyes, the epithelium was stratified squamous nonkeratinized for all the days. The stroma was composed of parallel collagen bundles with flattened keratocytes and the endothelium was simple squamous. There were no time-dependent changes through the experimental period (Figures $4 a-d$ ).
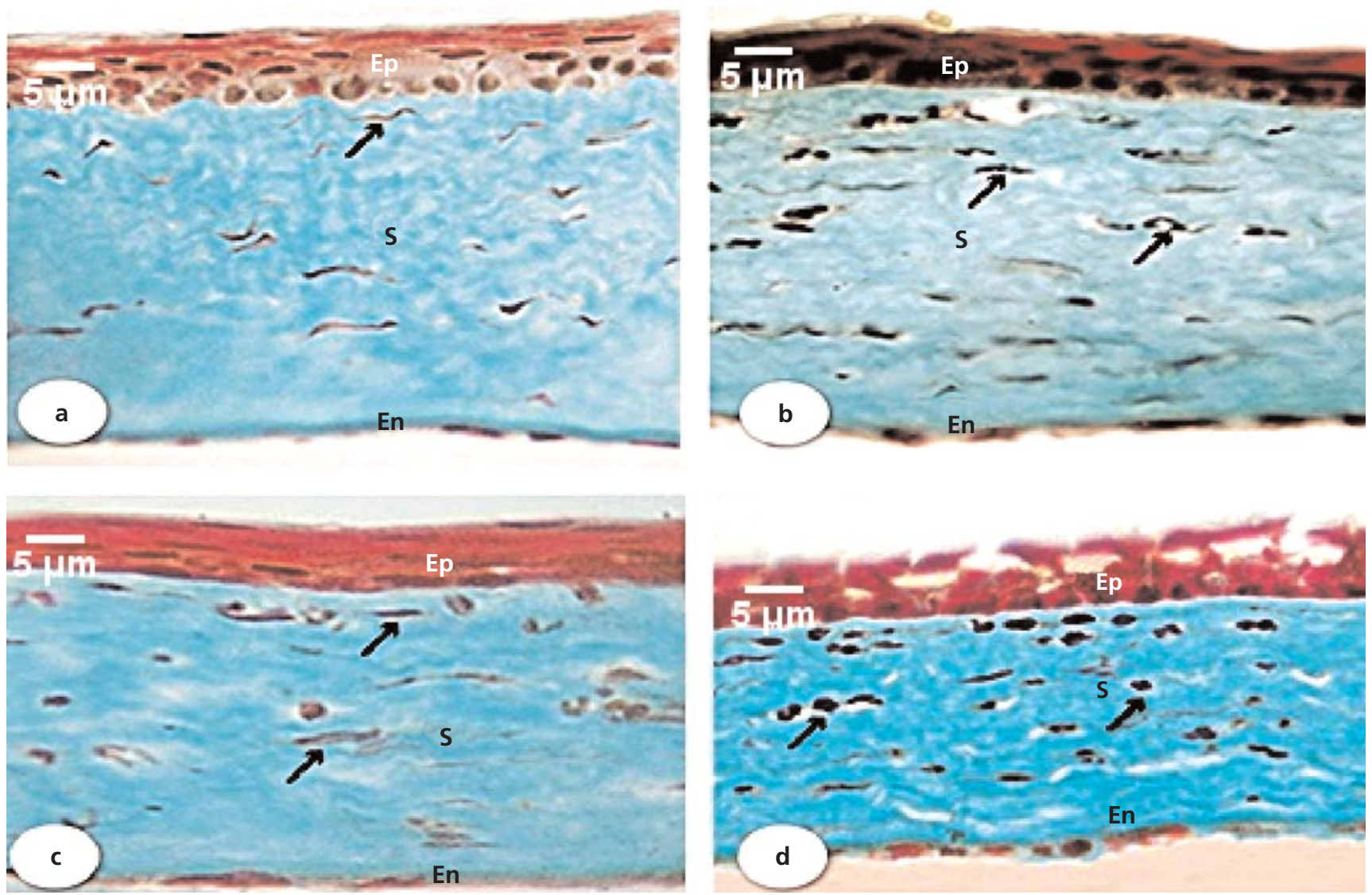

Figure 2. The photomicrographs of the cornea in the tarsorrhaphy group at day 5 (a), 10 (b), 15 (c) and 20 (d). Notice the gradual decrease in stratification of the corneal epithelium (Ep), increase in distribution of keratocytes in the stroma (S) and change in endothelial (En) cells size. The arrows indicate keratocytes (Masson's trichrome stain, $\times 400$ ). [Color figure can be viewed in the online issue, which is available at www.anatomy.org.tr] 

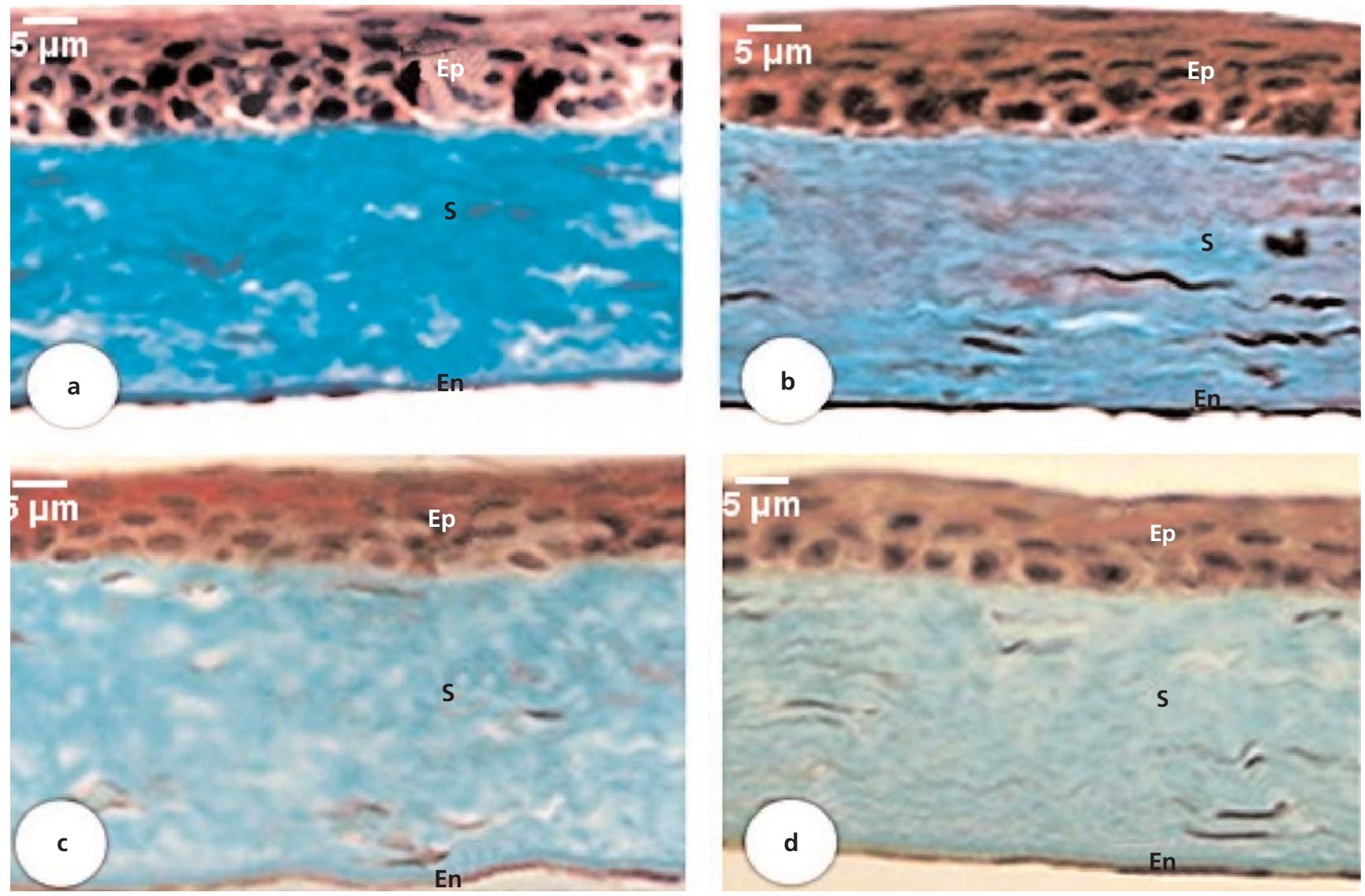

Figure 3. The photomicrographs of the cornea in the non-tarsorrhaphy (contralateral) eyes at day 5 (a), 10 (b), 15 (c) and 20 (d). En: endothelial cells, Ep: corneal epithelium, S: stroma. The organization is similar to the baseline (Masson's trichrome stain, $\times 400$ ). [Color figure can be viewed in the online issue, which is available at www.anatomy.org.tr]

\section{Discussion}

The current study demonstrated time-dependent changes in the closed eyes, with no light microscopic changes observed in the contralateral and control eyes.

The decrease in stratification of the corneal epithelium in the tarsorrhaphy eyes is in accordance with the study of Ladage et al. (2003) who reported a decrease in bromodeoxyuridine (BrdU) labelled corneal epithelial cells in the closed eye after eight days, signifying a decrease in proliferation over time. ${ }^{[10]}$ However, Yamamoto et al. ${ }^{[1]}$ who performed eyelid closure for 24 hours reported a decrease in surface cell exfoliation in the closed eye. They postulated that the absence of shearing forces of eyelid blinking in the closed eye state is protective over the surface epithelial cells. The discordance with the current study could be due to the very short period of eyelid closure in their studies. Adequate oxygen is a necessary factor in the highly regulated process of corneal epithelial renewal. ${ }^{[10]}$ Pérez et al. ${ }^{[12]}$ postulated that low oxygen levels suppress the aerobic epithelial metabolism which in turn affects cell renewal. The epithelial thinning and cell loss seen in the tarsorrhaphy eyes in the present study could therefore be due to an imbalance in the maintenance of the corneal epithelium secondary to the hypoxia induced by the eyelid closure.

The epithelial vacuolation observed in the tarsorrhaphy group at day 20 may possibly be a reflection of paraptosis or type III cytoplasmic cell death. ${ }^{[13]}$ This is a form of programmed cell death that is triggered by tumor necrosis factor (TNF) or insulin-like growth factor (IGF) ${ }^{[14]}$ It is characterized by cytoplasmic reorganization, enlargement of the endoplasmic reticulum and mitochondria, swelling of the cell and formation of extensive cytoplasmic vacuoles. ${ }^{[15]}$ In the eye, paraptosis has been portrayed in retinal ganglion cells following glaucoma induced retinal ischemia/reperfusion injury, a major cause of vision loss in the human. ${ }^{[16,17]}$ In addition, 

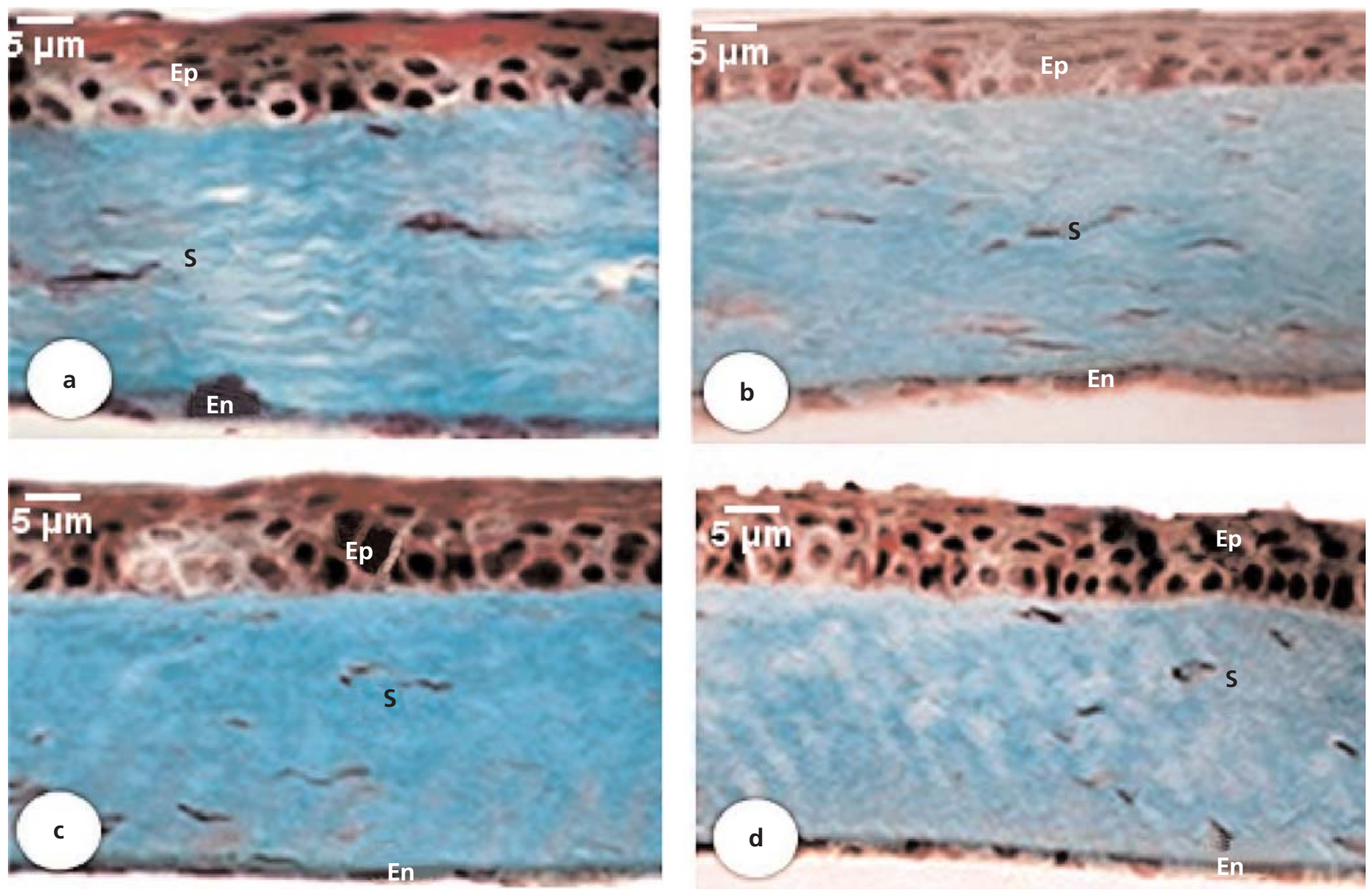

Figure 4. The photomicrographs of the cornea in the control group at day 5 (a), 10 (b), 15 (c) and 20 (d). En: endothelial cells, Ep: corneal epithelium, S: stroma. The organization is similar to the baseline (Masson's trichrome stain, $\times 400$ ). [Color figure can be viewed in the online issue, which is available at www.anatomy. org.tr]

paraptosis in the corneal epithelium may occur in pathological conditions such as corneal dystrophies. ${ }^{[18]}$

The stratification of the epithelium and presence of tight junctions in addition to other junctional complexes confer the barrier function to the cornea. ${ }^{[19]}$ Therefore, any perturbation of the corneal surface such as thinning compromises the integrity of this barrier and increases risks of mechanical damage, entry of microorganisms with subsequent occurrence of corneal diseases. ${ }^{[20]}$

The stromal keratocytes in the current study increased in distribution along with a change in shape in the tarsorrhaphy eyes, while the non tarsorrhaphy eyes had no obvious changes. The keratocytes are fibroblast like cells of the corneal stroma responsible for general repair and maintenance of the stromal components. They are normally in their quiescent stage and come into play only when there is a need for repair. ${ }^{[21]}$ The proliferation of the cells in the experimental group is postulated to be a response to the interruption of collagen fibers and extracellular matrix components as a result of corneal edema. When eyelid closure is prolonged, corneal metabolism is affected due to the resultant hypoxia. ${ }^{[2]}$ The anaerobic respiration that ensues, increases stromal acidosis which causes increased water imbibition into the stroma, hence edema. Another probable explanation is corneal epithelial - keratocytes interaction. The response of keratocytes can be influenced by cues from the overlying epithelium. ${ }^{[23]}$ The changes observed in the corneal epithelium may have caused these cells to release factors such as interleukin 1 which influenced the proliferation of the keratocytes. Increase in the number of keratocytes may be detrimental to the functioning of the cornea. Firstly, increase in keratocytes results in increased synthesis of stromal collagen fibers which may be irregular, resulting in hazy vision. ${ }^{[2,25]}$ Secondly, increased density of stromal keratocytes may lead to light scattering hence affecting the clarity of vision. ${ }^{[26]}$ The change in shape of the keratocytes in the 
tarsorrhaphy eyes also render them very efficient light scatterers. ${ }^{[26]}$ From this, it can be concluded that changes in keratocytes affect the light transmission ability of the corneal stroma.

In the current study, there was an apparent enlargement of corneal endothelial cells in the tarsorrhaphy eyes. Similar finding has been reported after prolonged low oxygen permeable contact lens wear on the corneal endothelium. ${ }^{[27]}$ Endothelial polymegathism which refers to variation in cell sizes of the endothelial cells has also been elucidated by the induced hypoxia. ${ }^{[28]}$

In this study, there were no apparent changes in the structure of the cornea in the control animals and the contralateral eyes in the subject animals. We postulate that this could be due to adequate atmospheric oxygenation or the effect could be minimal at light microscopic evaluation.

\section{Conclusion}

This study demonstrated degenerative changes in the tarsorrhaphy eye that can be deleterious to the functioning of the cornea. Therefore, it is recommended that tarsorrhaphy should not be prolonged due to risks for corneal diseases and diminution of vision as a result of the structural changes leading to reduced corneal transparency.

\section{Acknowledgement}

The authors are grateful to all teaching and technical staff of the University of Nairobi Departments of Human Anatomy and Biochemistry for their unwavering support.

\section{References}

1. Qazi Y, Wong G, Monson B, Stringham J, Ambati BK. Corneal transparency: genesis, maintenance and dysfunction. Brain Res Bull 2010;81:198-210.

2. Chhabra M. Oxygen transport through soft contact lens and cornea: lens characterization and metabolic modeling. ProQuest dissertations and theses 2007;69-03:1776.

3. Beebe DC. Maintaining transparency: a review of developmental physiology and pathophysiology of two avascular tissues. Semin Cell Dev Biol 2008;19:125-33.

4. Chhabra M, Prausnitz JM, Radke CJ. Modeling corneal metabolism and oxygen transport during contact lens wear. Optom Vis Sci 2009;86:454-66.

5. Cosar CB, Cohen EJ, Rapuano CJ, Maus M, Penne RP, Flanagan JC, Laibson PR. Tarsorrhaphy: clinical experience from a cornea practice. Cornea 2001;20:787-91.

6. Baum JL. The Castroviejo Lecture. Prolonged eyelid closure is a risk to the cornea. Cornea 1997;16:602-11.
7. Labbé A, Liang H, Martin C, Brignole-Baudouin F, Warnet JM, Baudouin C. Comparative anatomy of laboratory animal corneas with a new-generation high-resolution in vivo confocal microscope. Curr Eye Res 2006;31:501-9.

8. Williams DL. Ocular disease in rats: a review. Vet Ophthalmol 2002;5:183-91.

9. Tachibana M, Kasukabe, T, Kobayashi Y, Suzuki T, Kinoshita S, Matsushima Y. Expression of estrogen receptor alpha and beta in the mouse cornea. Invest Ophthalmol Vis Sci 2000;41:668-70.

10. Ladage PM, Ren DH, Petroll WM, Jester JV, Bergmanson JP, Cavanagh HD. Effects of eyelid closure and disposable and silicone hydrogel extended contact lens wear on rabbit corneal epithelial proliferation. Invest Ophthalmol Vis Sci 2003;44:1843-9.

11. Yamamoto K, Ladage PM, Ren DH, Li L, Petroll WM, Jester JV, Cavanagh HD. Effect of eyelid closure and overnight contact lens wear on viability of surface epithelial cells in rabbit cornea. Cornea 2002;21:85-90.

12. Pérez JG, Méijome JM, Jalbert I, Sweeney DF, Erickson P. Corneal epithelial thinning profile induced by long-term wear of hydrogel lenses. Cornea 2003;22:304-7.

13. Allen TC, Cagle PT. Basic concepts of molecular pathology. Arch Pathol Lab Med 2008;132:1551-6.

14. Sperandio S, Poksay K, de Belle I, Lafuente MJ, Liu B, Nasir J, Bredesen DE. Paraptosis: mediation by MAP kinases and inhibition by AIP-1/Alix. Cell Death Differ 2004;11:1066-75.

15. Hoa N, Myers MP, Douglass TG, Zhang JG, Delgado C, Driggers L, Callahan LL, VanDeusen G, Pham JT, Bhakta N, Ge L, Jadus MR. Molecular mechanisms of paraptosis induction: Implications for a non-genetically modified tumor vaccine. PLoS One 2009;4:e4631.

16. Wang Y, Xu K, Zhang H, Zhao J, Zhu X, Wang Y, Wu R. Retinal ganglion cell death is triggered by paraptosis via reactive oxygen species production: a brief literature review presenting a novel hypothesis in glaucoma pathology. Mol Med Rep 2014;10:117983.

17. Wei T, Kang Q, Ma B, Gao S, Li X, Liu Y. Activation of autophagy and paraptosis in retinal ganglion cells after retinal ischemia and reperfusion injury in rats. Exp Ther Med 2015;9: 476-82.

18. Benitez-del-Castillo JM, Lemp MA. Ocular surface disorders. London: JP Medical Publishers; 2013. p. 157-60.

19. Langbein L, Grund C, Kuhn C, Praetzel S, Kartenbeck J, Brandner JM, Moll I, Franke WW. Tight junctions and compositionally related junctional structures in mammalian stratified epithelia and cell cultures derived therefrom. Eur J Cell Biol 2002; 81:419-35.

20. Bennett ES, Weissman BA. Clinical contact lens practice. Philadelphia (PA): Lippincott Williams \& Wilkins; 2005. p. 11-4.

21. West-Mays JA, Dwivedi DJ. The keratocyte: corneal stromal cell with variable repair phenotypes. Int J Biochem Cell 2006;38:162531.

22. Edelhauser HF. The balance between corneal transparency and edema. The Proctor lecture. Invest Ophtalmol Vis Sci 2006;47: 1754-67.

23. Wilson SE, Liu JJ, Mohan RR. Stromal-epithelial interactions in the cornea. Prog Retin Eye Res 1999;18:293-309. 
24. Yanoff M, Duker JS. Ophthalmology. 3rd ed. St Louis (MO): Mosby; 2008. p. 362.

25. Meek KM, Leonard DW, Connon CJ, Dennis S, Khan S. Transparency, swelling and scarring in the corneal stroma. Eye (Lond) 2003;17:927-36.

26. Moller-Pedersen T, Cavanagh HD, Petroll WM, Jester JV. Stromal wound healing explains refractive instability and haze development after photorefractive keratectomy: a 1-year confocal microscopic study. Ophthalmology 2000;107:1235-45.

27. Magdum RM, Mutha N, Maheshgauri R. A study of corneal endothelial changes in soft contact lens wearers using non-contact specular microscopy. Medical Journal of Dr. D. Y. Patil University 2013;6:245-9.

28. Chaudhuri Z, Vanathi M. Postgraduate ophthalmology. Vol 1. New Delhi: Jaypee Brothers Medical Publishers; 2012. p. 100.

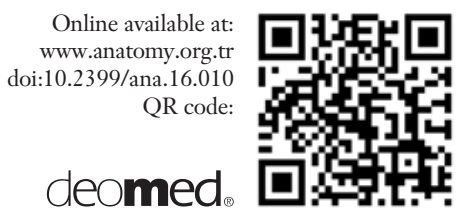

Correspondence to: Faith Bonareri Aseta, BSc

Department of Human Anatomy, School of Medicine,

Nairobi University, Nairobi, Kenya

Phone: +254 703643913

e-mail: bonareri.aseta@gmail.com

Conflict of interest statement: No conflicts declared.

\begin{abstract}
This is an open access article distributed under the terms of the Creative Commons Attribution-NonCommercial-NoDerivs 3.0 Unported (CC BY-NCND3.0) Licence (http://creativecommons.org/licenses/by-nc-nd/3.0/) which permits unrestricted noncommercial use, distribution, and reproduction in any medium, provided the original work is properly cited. Please cite this article as: Aseta FB, Mwachaka PM, Odula PO, Malek AK. Histomorphological changes in the cornea of the rat following monocular eyelid closure. Anatomy 2016;10(2):87-93.
\end{abstract}

